\title{
The effect of semantic priming on the detection of letters within words
}

\author{
TONI L. BLUM and NEAL F. JOHNSON \\ Ohio State University, Columbus, Ohio
}

\begin{abstract}
Smith (1979) reported an experiment in which subjects were to detect whether or not a displayed word contained a particular target letter. Her data indicated that if the word bearing the target letter was preceded by a semantically related item, the detection of the target letter was faster than it was if the preceding item was unrelated. Those results provided strong support for holistic models of word recognition, in which it is assumed that letter detection must be mediated by prior word recognition. That is, any facilitating effect of the prime on lexical access should be passed on to subsequent letter detection. The present experimental paradigm, which was very similar to (albeit different from) that of Smith, served to explore the generality of her effect, but the results did not confirm her findings. Although a lexical-decision task used in Experiments 2,5 , and 7 provided clear evidence that the priming items employed in these experiments did facilitate lexical processing, a letter-detection task used in Experiments 1, 3, 4, 6, and 8 failed to reveal any facilitating effect of semantic priming on letter detection. The conclusion is that the generality of Smith's effect is far too limited to offer support for holistic models of word recognition.
\end{abstract}

Holistic models of word recognition (e.g., that of Johnson, 1977) reject the notion that component-level cognitive processing necessarily mediates the identification of a word. Such models do not assume any initial cognitive processing at the component level, and they assume that cognitive encodings of letter-level information are not immediately available for use in any decision-making process. In fact, although various models differ as to whether they assume that holistic encoding always occurs (Johnson, 1977) or occurs only under some circumstances (Healy \& Drewnowski, 1983), they seem to agree on the assumption that if holistic encoding does occur, letter information must be derived from the word-level encoding (Johnson, Allen, \& Strand, 1989), rather than the other way around.

The key suggestion in these models is that, under normal circumstances, a usable cognitive encoding becomes available only at the pattern or word level of processing. This assumption is an integral part of the pattern-unit model (Johnson, 1975, 1977, 1979, 1981; Johnson, Turner-Lyga, \& Pettegrew, 1986), but it is also an important component of both Johnston and McClelland's (1980) hierarchical model and Healy and Drewnowski's (1983) unitization model.

An important implication of holistic views of word recognition is that the cognitive encoding of word-level information precedes that of letter-level information. That

Experiments 1, 2, 3, 5, and 6 in this study formed T.L.B.'s masters thesis, which was submitted to Ohio State University under the name of T. L. Strand. We are grateful for very helpful comments by Robert Proctor, Alice Healy, and two anonymous reviewers. Correspondence should be addressed to N. F. Johnson, Department of Psychology, Townshend Hall, Ohio State University, Columbus, $\mathrm{OH} 43210$. is, these views imply that although letter-level encoding should not mediate word-level encoding when the word is encoded holistically, a strong prediction is that under such circumstances (i.e., holistic encoding) word-level encoding must mediate letter detection.

If this is the case, anything that would facilitate wordlevel processing should also facilitate any subsequent letter-level processing. In support of this idea, Johnson et al. (1989) report an experiment in which word frequency was varied, and the subjects' task was to determine whether a displayed word contained a specific predesignated target letter. Consistent with Healy and Drewnowski's (1983) unitization theory, as word frequency increased there was an increasing concealment of the to-be-detected letter within the word. However, more critical to the present issue was that once word frequency was sufficiently great to ensure that the displayed word would be encoded holistically, increases in word frequency beyond that point resulted in a reduction in the latency for letter detection. That is, the facilitating effect of word frequency on word-level processing seemed to be passed on to the subsequent letter-level processing.

An experiment by Smith (1979) also offers data relevant to this issue. She used a semantic-priming paradigm, coupled with a letter-detection task, to determine the effect of the availability of lexical information on the detection of component information. She assumed that the priming of a target word with a semantically related word would facilitate lexical processing (Neely, 1976, 1977), and that if such processing must precede letter identification, it also should facilitate letter detection.

The prime words in Smith's (1979) task were identical to, semantically related to, or unrelated to the target words. The subjects were presented with an isolated prime 
word, which they read silently, followed by a target word, and the subjects' task was to determine whether the target word contained a particular target letter. The critical target letter for which they were to search appeared immediately above each letter position in the word when it was presented.

Smith (1979) found that when subjects were presented with a prime that was either identical to the target or semantically related to the target, they exhibited shorter latencies in letter detection than they did when presented with an unrelated prime word. The fact that letter detection was facilitated by priming of the word that contained the letter offers clear support for the idea that word-level encoding precedes the availability of letter-level information.

As has been noted, some views of holistic encoding (e.g., Johnson, 1977) make a strong statement regarding the assumption that word-level encoding must precede letter-level encoding, and that letter-level encoding is dependent on prior word-level encoding. That fact made it seem prudent to explore the effect reported by Smith (1979) in somewhat more detail. In particular, the generality of the effect is important, because some views suggest that it should always occur.

\section{EXPERIMENT 1}

The first experiment was designed to reestablish Smith's (1979) original finding that the facilitation of lexical processing does lead to the facilitation of letter detection. Specifically, the intent was to reproduce Smith's effect in the context of the particular experimental paradigm that has been used previously to explore both the pattern-unit and the unitization models (Johnson, 1977, 1981; Johnson et al., 1989).

In addition, it is clear that when subjects are asked to explicitly manipulate the information in a display in some manner (e.g., to detect a letter or make a lexical decision), the extra information processing acts as an overlay on what occurs normally during passive reading. As such, the processing itself can have distracting effects on the processing of subsequent stimuli (e.g., how a prime is processed can influence the subsequent processing of the target) (Kaye \& Brown, 1985; Smith, Theodor, \& Franklin, 1983). For that reason, as well as the desire to closely match the conditions used by Smith (1979), these experiments all involved the type of priming task used by Smith in which the prime was read passively, and not responded to in any specific manner.

\footnotetext{
Method

Apparatus. The apparatus included a Northstar Horizon computer and two Televideo $920 \mathrm{C}$ terminals. The experimenter controlled the course of the experiment by using one terminal, while the subject responded by using the other terminal. The displays, which appeared at the center of the subjects' screen, consisted of white characters on a dark background, with each character occupying a visual angle of approximately $.25^{\circ}$. The subjects responded by pressing the "Z"' key for "No" and the "?-l" key for "Yes."
}

Materials. The words were typed in uppercase letters, and they were presented in a single block of 180 displays. For each of the 180 display words, there existed a superordinate related-prime, and each of the 29 superordinates used in the experiment, as well as the 180 subordinate target display words, was chosen from the Battig and Montague (1969) category norms. Care was taken to ensure that the target display words occurred frequently (within the top 20 answers) of their corresponding superordinates, but neither word frequency nor word length was controlled.

To create the unrelated display pairs, the superordinate prime words were randomly re-paired with other target displays, and they were then evaluated by two independent judges to ensure that the primes were unrelated to their target displays. A control condition was also included, in which the prime was not a word but a string of question marks. The subjects received a related prime word for one third of the trials, an unrelated prime word for another third, and a control prime for the remaining third.

The first letter of each target display word was chosen to be the target letter for that item, and the subjects were informed of that fact. The only stipulation was that the target letter could not also appear in the prime word. The target-absent letters (foils) did not appear in either the prime or the target display words, and targetpresent trials and target-absent trials occurred equally often

Subjects. The subjects were 48 undergraduate students who participated as part of a course option. They were fully informed of the sequence of the display words, but not of potential relationships between them. Each subject received all 180 trials.

Procedures. Prior to each display, the subjects saw an " $X$ " centered on the video screen. At the same time, the target or foil letter appeared at the center of the experimenter's screen, but the subject did not see that display. The experimenter then stated the letter aloud and immediately pressed the carriage return. At this prompt from the experimenter's keyboard, the " $X$ " on the subject's screen was replaced with the prime (related, unrelated, or control), also centered on the screen. The prime appeared for $600 \mathrm{msec}$, at which time it was replaced by a string of " $\mathrm{X}$ "'s, acting as a masking stimulus, and the "X"s remained on the screen for 875 msec. The mask was then replaced by the target display word itself.

The subjects were instructed to view the display and then indicate, as quickly and accurately as possible, the presence or absence of the target letter in the initial position of the target word. The subjects indicated their responses by pressing a key marked "yes" with the right hand or the key marked "no" with the left hand. The target word remained on the screen until shortly after the key was pressed, at which time it was replaced by a feedback message indicating whether the subject was correct or incorrect.

The six display conditions (related, unrelated, and neutral primes, and target-present and target-absent trials) occurred randomly within the list of 180 displays, and across subjects each word appeared in each condition equally often. Six lists were used for the counterbalancing, and 8 subjects were randomly assigned to each list. All reaction times below $200 \mathrm{msec}$ or above $1,600 \mathrm{msec}$ were removed from the analysis, and only the latencies for correct responses were included.

\section{Results}

Error data. The error rates for Experiment 1 are presented in Table 1, and the overall error rate was $5.6 \%$. An analysis of the data indicated no effect of either response type or priming condition, and there was no interaction $(F<1.00$ in each case).

Latency data. The mean reaction time for each condition is presented in Table 1 . An overall analysis indicated a significant effect of response type (yes vs. no) $[F(1,47)$ 
Table 1

Latency Data in Milliseconds and Error Percentages for Experiment 1

\begin{tabular}{|c|c|c|c|c|c|c|c|c|}
\hline & \multicolumn{2}{|c|}{ Related Prime } & \multicolumn{2}{|c|}{ Unrelated Prime } & \multicolumn{2}{|c|}{ Control Prime } & \multicolumn{2}{|c|}{$M$} \\
\hline & RT & $\%$ Error & RT & $\%$ Error & RT & $\%$ Error & $\mathrm{RT}$ & $\%$ Error \\
\hline Yes & 557 & 5.14 & 555 & 6.24 & 568 & 5.90 & 560 & 5.76 \\
\hline No & 613 & 5.34 & 622 & 4.93 & 629 & 6.11 & 621 & 5.46 \\
\hline$M$ & 585 & 5.24 & 588 & 5.58 & 599 & 6.00 & 591 & 5.61 \\
\hline
\end{tabular}

$=174.85, p<.001]$ and priming condition $[F(2,94)=$ $7.67, p<.01]$, but no interaction $(F<1.00)$. A separate analysis indicated no difference between the related-prime and unrelated-prime conditions $(F<1.00)$, and the overall effect of priming seemed to be due to the control condition's eliciting slower reaction times than those in either of the other two conditions.

\section{Discussion}

The main effect of response type was expected, and it reflects the typical fast-yes outcome. Contrary to expectations, however, no difference was found in response latency between the related-prime and the unrelated-prime conditions. A glance at Table 1 suggests that the main effect for priming condition was due primarily to the fact that subjects were slower to respond to a target word when it was preceded by a control prime than when it was preceded by either a related prime (by $14 \mathrm{msec}$ ) or an unrelated prime (by $11 \mathrm{msec}$ ). In general, then, these data do not support the finding reported by Smith (1979) that the detection of a letter within a word is facilitated if the word itself is primed by the prior presentation of a related word.

\section{EXPERIMENT 2}

The crucial result to be explained is that no difference was found between the related-prime and unrelated-prime conditions. One possibility is that the related-prime words chosen for Experiment 1 were simply not effective in facilitating the lexical processing of the target word. To explore that possibility, a simple lexical-decision task was used in Experiment 2. If the related primes do facilitate lexical processing, decision times should be faster if the words are preceded by a related prime.

\section{Method}

Apparatus and Materials. The apparatus and stimuli for Experiment 2 were identical to those in Experiment 1, except that the control-prime condition was eliminated, and 60 pronounceable nonwords were added. The nonwords were unrelated to the words. The trials were constructed in such a way that a related-prime and target word appeared on 60 trials, an unrelated-prime and target word appeared on 60 trials, and a word prime that was followed by a nonword target display appeared on the remaining 60 trials.

Subjects. The subjects were 12 undergraduate students who participated as part of a course option.

Procedures. The experimenter initiated each trial with the press of a key, as in Experiment 1. The displays also appeared as in Experiment 1 , and whether a particular target word was preceded by a related or unrelated prime was counterbalanced across subjects.
The subjects' task in this experiment, however, was merely to indicate whether the target itself was a word or a nonword, and they responded by pressing keys marked "yes" and "no." "Yes" in this case indicated a word, whereas "no" indicated a nonword. All reaction times below $200 \mathrm{msec}$ or above $1,600 \mathrm{msec}$ were removed from the data, and only correct responses were included.

\section{Results and Discussion}

Error data. The error rates for related, unrelated, and nonword conditions were $1.68 \%, 3.77 \%$, and $5.98 \%$, for an overall rate of $3.81 \%$. The analysis of variance indicated a main effect of condition $[F(2,22)=7.05, p<$ .011 , but that outcome confounds prime condition with response type. A separate comparison of the data from word targets revealed that the related-prime condition resulted in reliably fewer errors than did the unrelated-prime condition $[F(1,11)=5.05, p<.05]$, but that the difference between the unrelated-prime condition and the nonword condition was not reliable $[F(1,11)=3.29, p>.05]$.

Latency data. The mean reaction times for the related, unrelated, and nonword conditions were 626,646 , and $718 \mathrm{msec}$, and the overall mean was $677 \mathrm{msec}$. The analysis of variance showed a significant effect for priming conditions $[F(2,22)=23.04, p<.001]$, but again that outcome confounds prime condition with response type.

A separate analysis comparing the related and unrelated prime conditions indicated a reliable priming effect $[F(1,11)=5.35, p<.05]$, with the latency for the related-prime condition being shorter than that for the unrelated-prime condition. Given these results, it is clear that the failure of the related prime to facilitate letter detection in the first experiment cannot be attributed to a failure of the related primes to facilitate lexical processing.

\section{EXPERIMENT 3}

Another possible explanation for the lack of a semanticpriming effect in letter detection centers on the fact that subjects were asked to search for the target letter only in the initial position of the target word. It may be that narrowing their focus in such a way enables them to extract the letter-level information before obtaining a wordlevel code. Although this concept presents a problem for some holistic models of word recognition (e.g., that of Johnson, 1977), it may be consistent with the data reported by Johnson and Blum (1988). They found that when subjects had a way of prefocusing their attention, the subjects were able to detect component letters of consonant arrays without being influenced by any initial attempts to encode the pattern as a whole, and Marmurek (1987) has 
Table 2

Latency Data in Milliseconds and Error Percentages for Experiment 3

\begin{tabular}{|c|c|c|c|c|c|c|}
\hline & \multicolumn{2}{|c|}{ Related Prime } & \multicolumn{2}{|c|}{ Unrelated Prime } & \multicolumn{2}{|c|}{$M$} \\
\hline & RT & \% Error & RT & $\%$ Error & RT & \% Error \\
\hline Yes & 628 & 8.05 & 635 & 8.19 & 632 & 8.12 \\
\hline No & 700 & 7.01 & 70 & & 702 & 6.91 \\
\hline$M$ & 664 & 7.52 & 670 & 7.50 & 667 & 7.51 \\
\hline
\end{tabular}

reported similar attentional effects. Experiment 3 was designed to eliminate the possibility of such a prefocusing strategy.

\section{Method}

Apparatus and Materials. The apparatus and stimuli were identical to those in Experiment 1, with the exception that the target letter could appear in any letter position of the target word, and the conditions with the question marks as the primes were not included. Target letters were chosen randomly from the target words, with the stipulation that the letter must not appear in the prime word.

Subjects. The subjects were 32 undergraduate students who participated as part of a course option.

Procedures. Again, the target or foil letter was presented verbally by the experimenter before the onset of each display, and the subjects signaled their responses in the same manner as they did for Experiment 1. Reaction times and errors were recorded, and mean reaction times for each subject for each condition were calculated after a trimming procedure identical to that used in the prior experiments.

\section{Results}

Error data. The error rates are presented in Table 2. The overall error rate was $7.5 \%$, but the analysis revealed no reliable effects of priming condition $(F<1.00)$ or response type $[F(1,31)=2.88, p>.05]$, nor was there a reliable interaction $(F<1.00)$.

Latency data. The mean reaction times are presented in Table 2 . The overall analysis of variance indicated a significant main effect for response type $[F(1,31)=$ $94.80, p<.001]$, with "yes" responses again being much faster than "no" responses. However, neither the main effect of priming condition $[F(1,31)=1.26, p>$ $.05]$ nor the response type $\times$ priming condition interaction $(F<1.00)$ was significant.

\section{Discussion}

As in Experiment 1, no differences were found between the related-prime and unrelated-prime conditions for letter-detection latencies. In that the subjects had no way of knowing in advance the letter position in which the target would appear (if it appeared), they could not prefocus their attention on a particular position. Therefore, al- though the subjects would have to process all of the information in the display, once again there was no evidence that the facilitation of lexical processing was passed on to letter detection.

\section{EXPERIMENT 4}

Another difference between Experiments 1 and 2 is that Experiment 2 included nonwords, and it is possible that the priming effects for words resulted from some type of contrast attributable to the presence of those items. To explore this issue, Experiment 4 was simply a replication of Experiment 3 with the addition of the nonword displays from Experiment 2. Again, the target letter could appear in any within-word position. The subjects were 36 students from the same population as that in the other experiments.

\section{Results}

Error data. The error data are presented in Table 3. The overall error rate was about $7 \%$, and the subjects did make more errors on the target-present trials $[F(1,35)=$ $5.14, p<.05]$. However, there was no effect of priming condition on the error rate $(F<1.00)$, and the interaction also was not reliable $(F<1.00)$. A separate analysis that included only the conditions with word stimuli also showed no effect of priming condition $(F<1.00)$ and no interaction $(F<1.00)$.

Latency data. The latency data are presented in Table 3. There was a reliable effect of both response type $[F(1,35)=136.73, p<.001]$ and display type $[F(2,70)$ $=10.65, p<.01]$, but the interaction was not significant $(F<1.00)$. However, the effect of display type was attributable to the slow responses to the nonwords, and when those items were removed from the analysis, the remaining 2-msec difference was both unreliable $(F<$ 1.00 ) and in the wrong direction.

\section{Discussion}

Once again, the data indicate that display conditions which offer clear evidence of facilitating lexical processing do not yield evidence that there is any facilitation of the detection of letters within the display. On the other hand, it is clear that the lexical status of the display did have an influence on reaction times. The subjects were much slower when the displays were nonwords than when they were words.

In some ways, this lexicality effect is a latency analogue of the word-superiority effect (Reicher, 1967; Wheeler, 1970), although it was not reflected in the error data. The latter point, along with the fact that the word-superiority

Table 3

Latency Data in Milliseconds and Error Percentages for Experiment 4

\begin{tabular}{|c|c|c|c|c|c|c|c|c|}
\hline & \multicolumn{2}{|c|}{ Related Prime } & \multicolumn{2}{|c|}{ Unrelated Prime } & \multicolumn{2}{|c|}{ Nonwords } & \multicolumn{2}{|c|}{$M$} \\
\hline & RT & $\%$ Error & RT & $\%$ Error & $\mathrm{RT}$ & \% Error & RT & $\%$ Error \\
\hline Yes & 597 & 7.33 & 596 & 8.19 & 625 & 7.81 & 606 & 7.78 \\
\hline No & 689 & 6.28 & 685 & 6.14 & 712 & 6.33 & 695 & 6.25 \\
\hline$M$ & 643 & 6.81 & 641 & 7.17 & 669 & 7.07 & 651 & 7.01 \\
\hline
\end{tabular}


effect is critically dependent on the nature of the masking conditions within the experiment (Johnston \& McClelland, 1980), suggests that despite some surface similarities, the phenomena that underlie these two effects may be quite different.

One possible interpretation of this latency-based lexicality effect is that subjects do in fact process the display as a whole before attempting to detect component information. However, if that is the case, letter detection should be influenced by both lexicality and the speed of wordlevel processing, but the data indicated no effect of wordlevel priming on letter detection.

An alternative interpretation is that perceivers begin processing the display as a whole as they are identifying the letters, and the increased difficulty of coping with a nonword as an integrated pattern detracts from the simultaneous letter-level processing. The latter account would explain why subjects are slower to respond to components of nonwords, while at the same time, except for extreme cases, the ease of processing words would not influence letter detection.

Finally, it is also possible that the advantage stems from a word advantage in very early perceptual processing. That is, the initial perceptual representation of the pattern in terms of either a word form or a nonword form (Schacter, 1992) would be much faster for the familiar words than the unfamiliar nonwords. Therefore, regardless of the nature of the subsequent task (i.e., letter detection or lexical decision), there would be a latency advantage for words over nonwords, but it might not reflect any differences in strategic cognitive processing.

\section{EXPERIMENT 5}

In Experiments 1, 3, and 4, the target/foil letters were presented verbally prior to the display. Because subjects heard the letter, they had to use some memory representation of the letter in the eventual detection task. Potentially, the simple act of holding a target letter in working memory throughout each display might interfere with the subjects' attention to, and processing of, the prime word itself. However, if that was the case, then holding a letter in memory also should interfere with the priming effect in a lexical-decision task.

\section{Method}

Experiment 5 was again a lexical-decision task, designed in much the same way as Experiment 2, with two exceptions: (1) Before each display, the subject heard a target letter; and (2) after making a lexical decision with the usual keypress, the subject indicated verbally the presence or absence of the target letter in the target word (or nonword). The target letter could appear in any position within the word.

The subjects were 12 undergraduate students who participated as part of a course option. They were instructed that the word/nonword decision was the primary task. In order to reduce interference as much as possible, the target word remained on the screen for
$600 \mathrm{msec}$ after the lexical decision was made, allowing time for the letter detection to occur after the primary task.

Reaction times were collected for the lexical-decision task only, and they were trimmed as for Experiment 2. Errors were recorded for both the lexical-decision task and the letter-detection task.

\section{Results}

Error data. Errors in letter detection were recorded only to ensure that the subjects did indeed hold the letter in memory with reasonable accuracy. The mean number of letter detection errors per subject was 3.83 , for an error rate of only $2 \%$.

The mean lexical-decision error rates for the related, unrelated, and nonword conditions were $6.27 \%, 6.24 \%$, and $10.69 \%$, for an overall rate of $7.73 \%$. The analysis showed a significant effect of conditions $[F(2,22)=5.12$, $p<.025$, but that outcome seems to reflect a word versus nonword difference, in that there was no real difference between the two priming conditions $(F<1.00)$.

Latency data. The mean reaction times for the related, unrelated, and nonword conditions were 849,881 , and $995 \mathrm{msec}$, and the overall mean latency was $908 \mathrm{msec}$. The analysis produced a significant effect for priming condition $[F(2,22)=27.23, p<.001]$, but again, that analysis confounded response type and priming condition. A separate comparison of the two word conditions showed that the subjects were indeed significantly faster in the related-prime condition than in the unrelated-prime condition $[F(1,11)=10.72, p<.01]$.

Additional analyses. In additional analyses, the two lexical decision experiments were compared as a betweensubject factor. The results indicated that there were significantly more errors in Experiment 5 than in Experiment 2 $[F(1,22)=10.47, p<.01]$ and that the subjects also exhibited reliably longer decision latencies in Experiment $5[F(1,22)=18.32, p<.001]$. No significant interactions emerged. Although these data would suggest that the subjects had more problems in this experiment, in which they had to hold a letter in memory, it must be kept in mind that the two experiments were conducted at different times, and that therefore they may not be directly comparable. The critical issue is the fact that the effect of experiment did not interact with any of the other effects.

\section{Discussion}

Although the additional analysis seems to indicate that holding a letter in memory may have made the entire task more difficult, there was no disruption of the facilitating effect of semantic priming. Again, when subjects made word-level decisions, the latencies were reliably shorter in the related-prime condition, just as in Experiment 2 (albeit in this experiment there were no related differences in error rate). These latency results, then, imply that the failure of a related prime to facilitate letter detection in Experiments 1, 3, and 4 cannot be explained in terms of interference from an extra item (i.e., a letter) being held in memory. 


\section{EXPERIMENT 6}

Smith's (1979) effect disappeared when the subjects were forced to search for a letter within the prime word as well as within the target. She suggested that the processing of the prime word in this condition did not occur at a semantic level, and that the prime therefore did not facilitate the processing of the target word.

It may be that in the present experiments the combination of presenting the target letter before the prime, as well as the focus on letter detection, induced subjects to process the prime in some nonsemantic manner. It is unclear why the presentation of the target letter did not seem to interfere with the processing of the prime in Experiment 5 , except that letter detection was not emphasized in that experiment. In any event, in Experiment 6 the prior presentation of the target/foil letter was eliminated, and the experiment more closely replicated the conditions of the Smith (1979) study in that the target letter was presented immediately above each letter in the display word.

\section{Method}

The apparatus and stimuli for Experiment 6 were the same as those for Experiment 3, and there were no nonword displays or neutral primes. The subjects were 32 undergraduate students who participated as part of a course option.

The display sequence was identical to that in Experiment 3, with the exception that the target/foil letter was not presented before the display. The prime appeared after the initial focal "X," replaced after $600 \mathrm{msec}$ by the array of "X"s (again $875 \mathrm{msec}$ ). The target word appeared immediately following the array of " $\mathrm{X}$ "s, with the target or foil letter being replicated above it in every letter position, as follows:

$$
\begin{array}{ll}
\text { GGGGG } & \text { FFFFF } \\
\text { TIGER } & \text { or } \\
\text { STUMP }
\end{array}
$$

As before, the program randomly selected the target letter from the target word, with the stipulation that the target letter could not appear in the prime word. In the event of a foil display (no item), the foil letter on the top line did not appear in either the prime or the target.

The four display conditions were presented randomly; and across subjects, each word appeared in the related prime and the unrelated prime conditions, as well as the two foil conditions, equally often. The subjects were instructed to view the display, and then to press the appropriate key as quickly and accurately as possible to indicate the presence or absence of the target letter within the display.

\section{Results}

Error data. The overall error rate was $9.7 \%$, and the rates for each condition are presented in Table 4 . There were no reliable effects of either priming condition $(F<$

Table 4

Latency Data in Milliseconds and Error Percentages

\begin{tabular}{|c|c|c|c|c|c|c|}
\hline & \multicolumn{2}{|c|}{ Related Prime } & \multicolumn{2}{|c|}{ Unrelated Prime } & \multicolumn{2}{|c|}{$M$} \\
\hline & RT & $\%$ Error & RT & $\%$ Error & RT & \% Error \\
\hline Yes & 816 & 10.35 & 822 & 10.27 & 819 & 10.31 \\
\hline No & 897 & 9.41 & 905 & 8.95 & 901 & 9.18 \\
\hline$M$ & 856 & 9.88 & 864 & 9.61 & 860 & 9.74 \\
\hline
\end{tabular}

for Experiment 6
$1.00)$ or response type $[F(1,31)=1.13]$, nor was the interaction significant $(F<1.00)$.

Latency data. The mean reaction times for each condition are reported in Table 4 . There was a significant effect of response type $[F(1,31)=49.81, p<.001]$, but neither the priming effect $[F(1,31)=1.19, p>.05]$ nor the interaction $(F<1.00)$ was significant.

\section{Discussion}

The error data indicate a rather high error rate, suggesting that, in general, this task may have been somewhat difficult for subjects, but there were no systematic effects of either independent variable. The latency data again revealed the typical fast-yes effect, but just as for Experiments 1, 3, and 4, there was no reliable effect of priming condition, even under these conditions that closely resembled those used by Smith (1979).

\section{EXPERIMENT 7}

The data for Experiments 7 and 8 were collected at the same time and subjects were assigned to each experiment in alternating order as they appeared for testing. A lexicaldecision task was employed in Experiment 7, and Experiment 8 involved a letter-detection task. The purpose of these two experiments was to approximate Smith's (1979) conditions as closely as possible, while at the same time ensuring that for the two tasks (i.e., lexical decision and letter detection), the materials, display characteristics, procedures, and subjects were as closely equated as the different task decisions would allow. In addition, it is important to note that the issue is not whether the Smith effect can be replicated, which is not the question, but rather the generality of the effect. That is, holistic models make the strong prediction that access to component information is mediated by access to an encoding of the pattern as a whole, and that any variable that facilitates patternlevel encoding also should facilitate component-level encoding. To the extent that there are task environments in which such component-level facilitation does not occur, models of that type will have difficulties.

\section{Method}

The subjects' task was to make a lexical decision for each of a series of letter arrays that appeared at the center of a monitor screen. The methods, materials, and procedures were the same as those in Experiment 2, with the single exception that replications of a single letter appeared immediately above each letter within a displayed item, just as in Experiment 6. For a random half of the displays, the replicated letter also appeared within the target display item, although that was of no consequence for this experiment. The subjects were 48 students from the same population as that used for the other experiments.

\section{Results and Discussion}

The overall error rate (percentage) was 4.5 , and for the related, unrelated, and nonword conditions the rates were $3.6,4.3$, and $5.4[F(2,94)=5.49, p<.05]$. A comparison of just the two types of word display indicated that the difference was not reliable $[F(1,47)=3.00, p>.05]$. 
The mean latencies for the three types of display were 555,571 , and $653 \mathrm{msec}[F(2,94)=134.25, p<.001]$, and an analysis of just the two word conditions indicated that the subjects were reliably faster when the target was related to the prime $[F(1,47)=14.95, p<.01]$. Consistently with the prior data, then, the data indicate that under these conditions which closely match those of Smith (1979), word-level processing was facilitated when a semantically related prime word appeared on the screen immediately before the critical target display. Once again, however, the critical theoretical issue is whether that word-level facilitation is passed on to letter-level processing.

\section{EXPERIMENT 8}

As noted above, the purpose of Experiment 8 was to determine whether the effect of word-level priming would be passed on to letter detection under conditions that closely matched both those used by Smith (1979) and those employed in the previous experiment. The subjects' task was to determine whether the letter that was duplicated in the line above the target word also appeared someplace within the word.

\section{Method}

The materials, display conditions, and procedures were identical to those used in Experiment 7, except for the fact that the subjects' task was to determine whether the letter on the top line appeared within the target display. When the target letter did appear in the displayed word it could be in any letter position, and it was selected randomly with the restriction that it could not have appeared within the prime. The subjects were 48 students from the same population as that used for the other experiments.

\section{Results and Discussion}

The error data are presented in Table 5. There was no reliable effect of display type $[F(2,94)=2.86, p>.05]$ or response type $(F<1.00)$, and the interaction too was not significant $[F(2,94)=1.50, p>.05]$. A similar analysis that included only the two types of word display also showed no effect of display type $[F(1,47)=2.96, p>$ $.05]$ or response type $[F(1,47)=1.63, p>.05]$ and no significant interaction $[F(1,47)=3.43, p>.05]$.

The latency data are presented in Table 5 . The overall effect of display type was significant $[F(2,94)=37.07$, $p<.01]$, as was the effect of response type $[F(1,47)=$ $181.74, p<.01]$, but the interaction was not reliable $[F(2,94)=1.51, p>.05]$.
Again, the main effect for display type seems to have been the result of long latencies for the nonwords, and a data analysis that involved only the two word displays confirmed that impression. Not only was there little difference between the related and unrelated priming conditions, but the $F$ was less than one $(F=.18)$. The interaction also was not reliable $[F(1,47)=3.06, p>.05]$, although there was the usual response-type effect $[F(1,47)$ $=160.11, p<.01]$. These data are consistent with those from all of the preceding experiments in indicating that the conditions that facilitate the word-level processing in a lexical-decision task do not necessarily facilitate the processing of the letters within the same words.

\section{CONCLUSIONS}

With regard to the priming effect reported by Smith (1979), it is important to note that none of these experiments are exact replications of that experiment, because they differ from hers in a number of important ways (e.g. . different materials, display conditions, and types of equipment). However, these data do indicate that the generality of the effect she reported is severely limited. Experiments 2,5 , and 7 indicate quite clearly that lexical processing was facilitated in these experiments, yet Experiments 1 , $3,4,6$, and 8 indicate that these effects were not passed on to the processing needed for letter detection. This was true despite the fact that some of the letter-detection experiments included four times as many subjects as did some of the lexical-decision experiments.

In addition, although the holding of a letter in memory, as required by the letter-detection experiments, did seem to modulate overall performance in the lexicaldecision task, the critical priming effect was not influenced by memory load. Furthermore, when memory load was eliminated in the letter-detection task (Experiments 6 and 8), there still was no evidence that any facilitation of lexical processing was passed on to letter detection.

There have been arguments that the lexical-decision task may reflect post-lexical-access phenomena (see, e.g., Balota \& Chumbley, 1990, for a recent review of the issue), and if this is the case, it is possible that the facilitation of lexical processing revealed in the second, fifth, and seventh experiments may actually reflect just a facilitation of some type of cognitive processing that occurs immediately subsequent to lexical access. On the other hand, although the effects of word frequency seem to be

Table 5

Latency Data in Milliseconds and Error Percentages for Experiment 8

\begin{tabular}{lcccccccc}
\hline & \multicolumn{2}{c}{ Related Prime } & \multicolumn{2}{c}{ Unrelated Prime } & \multicolumn{2}{c}{ Nonwords } & \multicolumn{2}{c}{$M$} \\
\cline { 2 - 9 } & RT & \% Error & RT & \% Error & RT & \% Error & RT & \% Error \\
\hline Yes & 838 & 11.06 & 849 & 10.96 & 890 & 13.13 & 859 & 11.72 \\
No & 945 & 11.15 & 940 & 13.69 & 990 & 13.04 & 958 & 12.63 \\
$M$ & 892 & 11.10 & 894 & 12.32 & 941 & 13.08 & 909 & 12.17 \\
\hline
\end{tabular}


ambiguous with respect to when they influence lexical processing (Balota \& Chumbley, 1984, 1990), there may be less ambiguity with regard to priming effects (Seidenberg, Walters, Sanders, \& Langer, 1984; although also see Chumbley \& Balota, 1984, as a counterexample).

However, these points notwithstanding, the type of complete word-level encoding that holistic models of word recognition assume must precede letter-level encoding would also include some postaccess attention-driven processing such as that described by Johnston and McClelland (1980). Therefore, regardless of where the facilitation occurs, such models would expect that anything apparent in the lexical-decision task should be passed on to letter detection.

Clearly, other processing assumptions might also predict that word priming should be passed on to letterlevel processing, but in these experiments, the critical issue was the other side of the coin (i.e., the problem created by the absence of priming effects). For this reason, the fact that a priming relationship that had a very marked effect on lexical decision had no effect on letter detection raises serious questions regarding any model that assumes that letter encoding can occur only after word-level processing is completed.

\section{REFERENCES}

Balota, D. A., \& Chumbley, J. I. (1984). Are lexical decisions a good measure of lexical access? The role of word frequency in the neglected decision stage. Joumal of Experimental Psychology: Human Perception \& Performance, $10,340-357$.

Balota, D. A., \& Chumbley, J. I. (1990). Where are the effects of frequency in visual word recognition tasks? Right where we said they were! Comment on Monsell, Doyle, and Haggard (1989). Journal of Experimental Psychology: General, 119, 231-237.

Battig, W. F., \& Montague, W. E. (1969). Category norms for verbal items in 56 categories: A replication and extension of the Connecticut category norms. Journal of Experimental Psychology Monographs, 80 (3, Part 2).

Chumbley, J. I., \& Balota, D. A. (1984). A word's meaning affects the decision in lexical decision. Memory \& Cognition, 12, 590-606.

HeAly, A. F., \& DrewnowsKI, A. (1983). Investigating the boundaries of reading units: Letter detection in misspelled words. Journal of Experimental Psychology: Human Perception \& Performance, 9 , 413-426.

JoHNSON, N. F. (1975). On the function of letters in word identification: Some data and a preliminary model. Journal of Verbal Learning \& Verbal Behavior, 14, 17-29.
JOHNSON, N. F. (1977). A pattern-unit-model of word identification. In D. LaBerge \& S. J. Samuels (Eds.), Basic processes in reading: Perception and comprehension (pp. 91-125). Hillsdale, NJ: Erlbaum.

Johnson, N. F. (1979). The role of letters in word identification: A test of the pattern-unit model. Memory \& Cognition, 7, 496-504.

Johnson, N. F. (1981). Integration processes in word identification. In O. J. L. Tzeng \& H. Singer (Eds.), Perception of print: Reading research in experimental psychology (pp. 29-63). Hillsdale, NJ: Erlbaum.

Johnson, N. F., Allen, P. A., \& Strand, T, L. (1989). On the role of word frequency in the detection of component letters. Memory \& Cognition, 17, 474-482.

Johnson, N. F., \& BLum, A. J. (1988). When redundancy hurts letter detection: An attempt to define one condition. Perception \& Psychophysics, 43, 147-155.

Johnson, N. F., Turner-Lyga, M. , Pettegrew, B. S. (1986). Partwhole relationships in the processing of small visual patterns. Memory \& Cognition, 14, 5-16.

Johnston, J. C., MCClelland, J. L. (1980). Experimental tests of a hierarchical model of word identification. Joumal of Verbal Leaming \& Verbal Behavior, 19, 503-524.

KAYE, D. B., \& BROWN, S. W. (1985). Levels and speed of processing effects on word analysis. Memory \& Cognition, 13, 425-434.

MarmureK, H. H. C. (1987). Aftentional holism in visual word processing. Psychological Research, 49, 45-52.

NeELY, J. H. (1976). Semantic priming and retrieval from lexical memory: Evidence for facilitatory and inhibitory processes. Memory \& Cognition, 4, 648-654.

NeeLY, J. H. (1977). Semantic priming and retrieval from lexical memory: Roles of spreading activation and limited-capacity attention. Journal of Experimental Psychology: General, 106, 226-254.

REICHER, G. M. (1967). Perceptual recognition as a function of meaningfulness of stimulus material. Joumal of Experimental Psychology, 81, 275-280.

SCHACTER, D. L. (1992). Understanding implicit memory: A cognitive neuroscience approach. American Psychologist, 47, 559-569.

Seidenberg, M. S., Walters, G. S., Sanders, M., \& Langer, P. (1984). Pre- and postlexical loci of contextual effects on word recognition. Memory \& Cognition, 12, 315-328.

Sмiтh, M. C. (1979). Contextual facilitation in a letter search task depends on how the prime is processed. Journal of Experimental Psychology: Human Perception \& Performance, 5, 239-251.

Smith, M. C., Theodor, L., Frankuin, P. E. (1983). The relationship between contextual facilitation and depth of processing. Journal of Experimental Psychology: Learning, Memory, \& Cognition, 9, 697-712.

Wheeler, D. D. (1970). Process in word recognition. Cognitive Psychology, 1, 59-85.

(Manuscript received July 20, 1990; revision accepted for publication October 8, 1992.) 